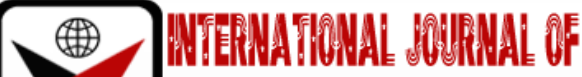

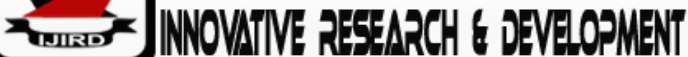

ISSN 2278 - 0211 (Online)

\section{Awareness, Availability and Accessibility of Pre-Exposure Prophylaxis (PREP) and Post Exposure Prophylaxis (PEP) in Health Institutions in Ekiti State, Nigeria}

Ekundayo O.K
Lecturer, Department of Biological Science,
Bamidele Olumilua University of Education Science and Technology, Nigeria
Ogundipe Love O.
Project Manager, Department of Public Health Consultant,
Environmental Development and Family Health Organization (EDFHO), Nigeria
Dr. Adeyanju Mary E.
Executive Director, Society for Women and AIDS in Africa, Nigeria (SWAAN), Nigeria
Omoniyi Olabimpe R.
Student, Department of Biological Science,
Kwara State University, Kwara State (KWASU), Nigeria

\begin{abstract}
:
The study was conducted to determine the level of awareness, availability and accessibility of both pre-exposure prophylaxis (PrEP) and post exposure prophylaxis (PEP) in health institutions in Ekiti State. Five major health facilities providing Antiretroviral Therapy (ART) cutting across the three senatorial districts of the state were purposefully selected based on number of patients accessing the facilities for different reasons and been a functional ART centre in the state. Key informant interview (KII) was employed to collect information from the respondents. The result shows that PrEP is not available with the state as none of the Centres visited had provided PrEP services in the last one year. Though PEP is available in all the facilities visited but accessibility is low. Mostly PEP is provided to Health Workers who got exposed in the course of their duty and victims of sexual violence (mostly rape). There is no comprehensive programing for discordant couples who could have benefited from PrEP programing. Awareness of PEP services where available is low thereby leading to low service uptake. Only one centre reported external access (an individual with accidental condom bust) to PEP over two years ago. Disclose is still a big issue within the state, most PLHIV have partners that are not aware of their status (Probably because of stigma) therefore creating a barrier for effective programing. Recommendations focused on the ways to address the issues raised in the result above.
\end{abstract}

Keywords: HIV (Human Immunodeficiency Virus), PrEP (Pre-exposure Prophylaxis), PEP, or Post-exposure Prophylaxis

\section{Introduction}

For the first years of the HIV epidemic, condom use was practically the only method available for preventing HIV transmission through sexual contact. In recent years, researchers have made considerable progress with alternative prevention methods, such as post-exposure prophylaxis (PEP) and pre-exposure prophylaxis (PrEP) (Curtis et al., 2016). Pre-exposure prophylaxis (or PrEP) is when people at very high risk for HIV take daily medicine to prevent HIV. PrEP involves a daily dose of antiretroviral drugs to reduce the likelihood of HIV transmission from subsequent exposures. To date, oral PrEP has only been approved in 34 countries globally. Kenya and South Africa are the two countries in subSaharan Africa with demonstration projects serving at-risk adults (PrEP Watch, 2016; Gilead, 2016; European Medicines Agency, 2016).Studies have shown that PrEP reduces the risk of getting HIV from sex by about $99 \%$ when taken daily. PrEP is recommended for populations at risk of HIV/AIDS to reduce HIV transmission. Studies suggest that it is highly effective for preventing HIV if it is used as prescribed (HIV and AIDS, 2017). While PrEP can provide very effective protection against HIV, it does not provide protection against other sexually transmitted infections (STIs) and blood-borne illnesses such as Hepatitis C, syphilis, and gonorrhea (WHO 2017). The effectiveness of PrEP is closely linked to adherence - if someone taking PrEP regularly misses some daily doses, their risk of HIV infection will increase substantially. It is therefore important that any programme offering PrEP advices on the importance of PrEP adherence.

PEP involves taking antiretroviral drugs after being exposed to HIV. This practice was initially evaluated in health care workers with occupational exposure to HIV. However, PEP is not always effective; it does not guarantee that someone exposed to HIV will not then become infected with HIV (Katzenstein et al., 2000). Of course, for these methods to have an 
impact on the HIV epidemic, people must be aware that they are available and use them as indicated. For example, for PEP to be effective, treatment must begin within 72 hours after exposure (Curtis et al., 2016). Similarly, to initiate PrEP, an individual must seek healthcare; therefore, awareness of these methods is an important first step in incorporating them into HIV prevention strategies.This study aimed at determining the level of awareness, availability and accessibility of PrEP and PEP in Ekiti State.

\section{Methods}

This study was conducted using a key informant interview with healthcare providers and ART centre personnel in five local government areas across the three senatorial districts of Ekiti State. The local governments include Ado, Emure, Ikere, Ido/Osi and Ise. Facilities used for the study were purposively selected based on number of clients accessing the facility and provision of ART services. The facilities include Federal Teaching Hospital Ido, Ekiti State University Teaching Hospital Ado, Okeisha Comprehensive Health Centre Ado, State Specialist Hospital Ikere, and Comprehensive Health Centre (Oke Yard) Ise.

Semi structured interviews were conducted with the ART focal person and other personnel at the centre to determine awareness, availability and accessibility of PrEP and PEP within the state.Each interview was tape recorded, transcribed and analyzed using quantitative data approach. The resulting issues were listed, discussed and recommendation for improvement provided.

\section{Ethical Consideration}

Ethical approval for the study was gotten from the Ekiti State AIDS Control Agency's technical committee on research.

\section{Results}

- PrEP is not available with the state as none of the Centres visited had provided PrEP services in the last one year.

- PEP is available in all the facilities visited but accessibility is low. Mostly PEP is provided to Health Workers who got exposed in the course of their duty and victims of sexual violence (mostly rape).

- There is no comprehensive programing for discordant couples who could have benefited from PrEP programing.

- Awareness of PEP services where available is low thereby leading to low service uptake. Only one centre reported external access (an individual with accidental condom bust) to PEP over two years ago.

- Disclose is still a big issue within the state, most PLHIV have partners that are not aware of their status (Probably because of stigma) therefore creating a barrier for effective programing.

\section{Discussion}

The findings of this study provide insights on the availability and accessibility of PrEP and PEP in various health institutions within Ekiti State.

PrEP is not being practiced in any of the health institutions as there is no policy guiding it or the policy is not yet implemented. This is a huge gap in HIV programing within the state with a high number of most at risk population. Most of the facility personnel were either not aware or do not understand the protocol for PrEP programing. This has a huge implication especially for PLHIV who are discordant couples and trying for children. Rather than encourage PrEP most facilities within the state uses of the book methods and theories for such couples, this includes;

- Encouraging couples to have sex without protection as long as the partner's viral load is below 20 copies

- When a female partner is the positive partner, the husband can use punctured condom which allow sperm flow to the virginal

- Option of sperm washing and artificial insemination is available but no couple have opted for it due to cost and cultural believes

- Working around the ovulation period to limit exposure when the partner's viral load is below 20 copies is also encouraged by some facilities.

The cost of implementing PrEP will be huge creating sustainability worries among stakeholders, it is important the state program make PrEP available for at least the most at risk populations.

Though PEP is available in all the facilities visited, it is mostly accessible for occupational exposure (health workers) and rape victims mostly referred by the police. Considering a lot of rape cases go unreported, awareness on PEP availability within the state is low leading to low service utilization. Despite the fact that PEP is available for the general population only two facilities recall providing PEP individuals (over two years ago) outside these categories. This is in line with the findings of Anthony et al., 2018 which measure use of PrEP and PEP among Nigeria University Student and reported low level of awareness of both prophylaxes. Therefore, there is urgent need to create awareness specifically on availability of PEP and the various situations that require PEP.

In addition, the study shows a high level of non-disclosure among PLHIV in the state creating barrier in effective couple/family management for service providers. Various interviews conducted reveal a need for updated knowledge and techniques for HIV counselors. Disclosure counselling seems to be limited to pre and posttest counselling period when patient is most likely still in denial while partners'tracing and couples' counselling seems to be lacking in practice.

For any gains in PrEP/PEP programing to be sustainable, disclosure barrier must be overcome. Follow up counseling and use of role models (PLHIV who have overcome disclosure barrier) will have great impart. This should be matched with awareness on stigma reduction and engagement of PLHIV as counselors with public health system in the state. 


\section{Conclusion}

PrEP and PEP can be important parts of public health efforts to prevent the spread of HIV with proper programing. Awareness of available services and community programing to overcome disclose barrier should be a priority. The outcome of various strategies deployed by facilities for discordant couples in the absence of PrEP should be a subject of further study.

\section{Recommendation}

- Integrated PrEP and PEP programing is required which must take in account capacity building for service providers to effectively communicate PrEP and PEP with clients

- Focused awareness on service availability should be included in larger HIV awareness program

- Training of counselors on improved counseling techniques and community of practice to encourage interchange of ideas should be promoted. This is of particular importance since continuous counselling is required for PLHIV

- Deliberate policy to engage PLHIV as counselors and promote role models to overcome disclosure barrier

\section{Acknowledgement}

The study acknowledges the contribution of the leadership of all ART Centres in Ekiti state and the cooperation of selected personnel in each of the facilities. The support of the Ekiti State AIDS Control Agency (EKSACA) to the success of the study is also acknowledged.

\section{References}

i. Curtis Dolezal, Timothy Frasca, Rebecca Giguere, Mobolajilbitoye, Ross D. Cranston, Irma Febo, Kenneth H. Mayer, Ian McGowan,and Alex Carballo-Diéguez. Awareness of post-exposure prophylaxis (PEP) and preexposure prophylaxis (PrEP) is low but interest is high among men engaging in condom less anal sex with men in Boston, Pittsburgh, and San Juan. 2016

ii. Gilead. European Commission Grants Marketing Authorization for Gilead's Once-Daily Truvada ${ }^{\circledR}$ For Reducing the Risk of Sexually Acquired HIV-1. Gilead Sciences, Inc; 2016

iii. Katzenstein TL, Dickmeiss E, Aladdin H, Hede A, Nielsen C, Nielsen H, Gerstoft J. Failure to develop HIV infection after receipt of HIV-contaminated blood and post exposure prophylaxis. Annals of Internal Medicine. 2000; 133(1):31-34.

iv. 'Men Who Have Sex with Men (MSM), HIV and AIDS.'AVERT. Web. Accessed 13 September 2017. $<$ https://www.avert.org/professionals/hiv-social-issues/key-affected-populations/men-sex-men>MSM.

v. World Health Organization. 'WHO implementation tool for pre-exposure prophylaxis (PrEP) of HIV infection'. 2017. 